\title{
Toward Anatomical Simulation for Breath Training in Mind/Body Medicine
}

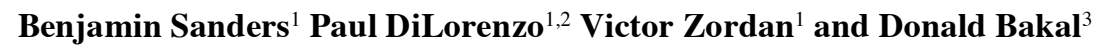

\begin{abstract}
The use of breath in healing is poorly understood by patients and professionals alike. Dysfunctional breathing is a characteristic of many unexplained symptoms and mind/body medical professionals seek methods for breath training to alleviate such problems. Our approach is to re-purpose and evolve a recently developed anatomically-inspired respiration simulation which was created for synthesizing motion in entertainment for the use of visualization in breath training. Our motivation comes in part at the request of medical experts who saw the value in such a tool for medical applications in modeling and visualizing physiology of respiration. In addition, in mind/body medicine, problems are often created from patients being advised to breath according to some standard based on pace or volume. However, a breathing pattern that is comfortable and effortless for one person may not have the same benefits for the next person. The breathing rhythm which is most effortless for each person needs to be dynamically identified. To this end, in this paper, we explore an adaptation method that employs optimization to modify a generic model of respiration to fit the breath patterns of specific individuals. Subjects are recorded using motion capture equipment and their motion data is used to derive simulation control parameters. In practice, the corresponding visualization which is specific to individual patients could to be used to train proper breath behavior, both by showing specific (abnormal) practice and recommended modification(s).
\end{abstract}

\section{Introduction}

The breath is the ultimate healing heuristic in mind/body medicine; yet the use of the breath in healing is poorly understood by patients, professionals and the public at large. In mind/body medicine, effortless breathing is a heuristic to guide patient development of somatic awareness in the management of medically unexplained symptoms (chronic fatigue, fibromyalgia, irritable bowel, non-cardiac chest pain). Dysfunctional breathing is a characteristic of the majority of cases related to these symptoms and therefore careful breath training is both critical but

\footnotetext{
${ }^{1}$ University of California, Riverside, CA, USA \{bsanders/pdiloren/vbz\}@cs.ucr.edu

${ }^{2}$ Dreamworks Animation SKG, Glendale, CA, USA

${ }^{3}$ University of Calgary, 2500 University Drive, Calgary, Canadadbakal@ucalgary.ca
} 
difficult to administer, in part because breathing is a highly individualized attribute that is under both conscious and unconscious control.

Our approach is to develop motion simulation of respiration which will be used for the purposes of educating and training patients. Visualization of the respiratory system is made possible through an anatomically inspired, physically based model of the torso. This model includes all of the key muscles, bones, and connective components found in the human trunk. We have developed this simulation for use in the synthesis of realistic breath motion for visualization of healthy and paradoxical breathing. A similar respiration system [6] is currently in place used as a tool for educating individuals about breath mechanics for stress management by trainers of Blue Cross but, in this effort, we extend the simulation with the goal of breath abnormalities heading toward the use of the simulation for breath training. In addition, a second goal is to modify the simulation to be most appropriate and realistic for specific patient breath training.

Motivating personalized models of breath respiration, in mind/body medicine, problems are often created from patients being advised to breath according to standard metrics, for example based on pace or volume. In practice, a breathing pattern that is comfortable and effortless for one person may not have the same benefits for the next person. The breathing rhythm which is most effortless for a single individual is derived from several factors, including quantifiable physical characteristics such as their stature, height, weight, and so on as well as from immeasurable quantities such as muscle features, voluntary habits that have become subconscious, and potentially, injuries and the corrections/allowances individuals have made for them over time. A breath signature needs to be dynamically identified for each person. To this end, we are proposing to explore adaptation methods that employ optimization techniques to modify a generic model of respiration to fit the breath patterns and characteristics of specific individuals. To accomplish this, patients would be recorded using state of the art optical-infrared motion capture equipment employing non-invasive motion markers at key landmarks on the body. The patient's own motion data will be used to derive motion control parameters. From this effort, a visualization that is specific to the patient will be used to train breathing for the individual, both by showing their common (abnormal) practice and recommended modifications.

\section{Related Work}

Visual and physical simulation of synthetic anatomical muscles has been described for several applications related to modeling and animation, for example in the head, neck and face [7-13], the hand [14-16], and for skeletal muscles [17-22]. Visual simulation of skeletal muscles has been approached procedurally through heuristic shape changes made in response to bone movement $[18,19,23]$. These 
examples model the change in shape of a muscle through geometric muscle bellies that stretch and deform based on length.

Physically based approaches for skeletal muscles include the early work of Chen and Zeltzer [17] who use a biologically based muscle model to generate proper muscle force and Teran and colleagues who use a finite volume method (FVM) to create a continuous internal-tension based muscle simulation focusing on the muscles of the arm [21, 22]. Both show results of deformation on the muscles systems of a single limb. In addition, Nedel and Thalmann propose the use of a spring-mass system as an alternative for real-time applications [20].

We have found no examples of work which are performed on active muscle deformation for animation of the human body, still fewer examples of the nature and complexity of those described here. Active deforming muscles yield the impetus for respiration physiology, namely the contraction of the diaphragm. Close to our efforts are the models of Kaye et al. [1] who animate deformable lungs for clinical applications based on CT scans and simplified cardiopulmonary mechanics and the constraint-based solver of Promayon et al. [2] which models the deformation of the abdomen during calm breath. However to the best of our knowledge, ours is the first work to investigate the animation of the torso by simulating the motion of both bone such as the ribcage and deformable subcomponents such as the abdomen.

\section{Motivation: Mind/Body Medicine}

Evidence demonstrates that stress may exacerbate or cause illness and that mind/body interventions can improve clinical outcomes. Mind/body medicine is a scientifically-validated medical discipline. Interventions, including cognitive/behavioral therapy, relaxation response training, lifestyle modifications addressing nutrition and exercise, and coping strategies, have been applied successfully to decrease the frequency and intensity of medical symptoms and to improve disease management in patients with hypertension, heart disease, chronic pain, insomnia, infertility, menopause and a host of other conditions with a stress component.

Medically unexplained symptoms account for over half of all outpatient visits with 20-25 percent remaining chronic and recurrent. Non-cardiac chest pain is estimated to account for up to 70 percent of all patient presentations with chest pain. With population growth and increasing case load pressure on medical specialists, it is clear that an improved method of patient management is required. Conventional pharmacological, psychological and alternative treatments (supplements) for medically unexplained symptoms are generally ineffective. Through training in recognition and practice of effortless breathing, patients and physicians will have the means to enhance tacit holistic knowing and self-management through the use of their bodies. 
In addition, capturing 'dysfunctional', 'normal' and 'effortless' breathing patterns is not readily achieved with current methodologies. The quantification of breathing is generally achieved through the use of strain gauge devices affixed to the abdomen and chest. These measures provide average data points which are used to make comparisons in terms of static volume differences and rate. These measures do not lend themselves to identifying the subtle motion that captures the actual movement of the upper chest, shoulders, thoracic and abdominal regions during inhalation and exhalation.

\section{Approach Overview}
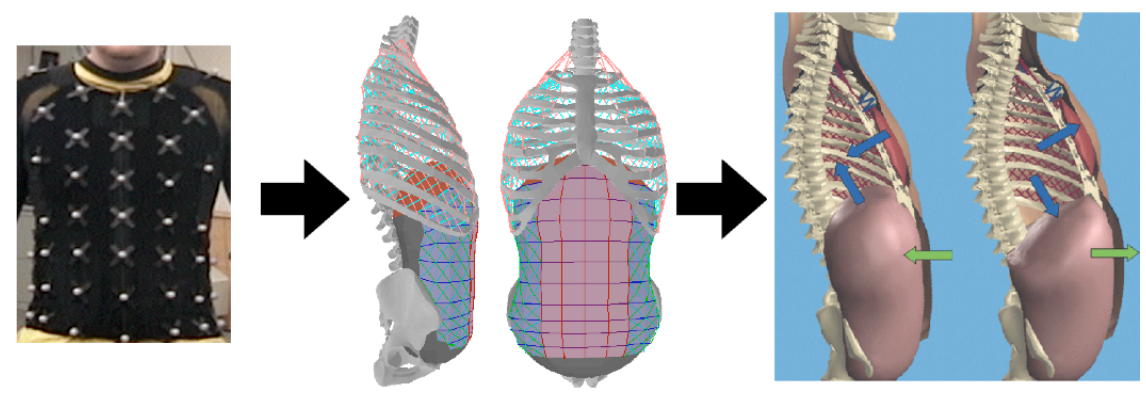

Fig. 1 Left: Marker data on the human torso is input. Center: A composite trunk simulation for breathing simulation, articulated rigid-body bones and deformable surfaces for the diaphragm and abdomen. Right: Through optimization, motion is synthesized for the trunk which matches the motion data.

We propose to use forward dynamic simulation in order to manage the inherent complexity of the processes as well as to increase the benefit of these efforts in fields beyond computer graphics - the dynamics lead to approximations of forces, accelerations, stresses, and other important factors that may be of interest to medical researchers. In computer animation, physical simulation has been used extensively to model rigid-body motion and deformation of bodies (separately), but our choice of modeling bodily function diverges from previous efforts because human physiology readily mixes rigid and flexible components requiring an inherently complex "mixed" simulation structure. We introduced the premiere physical model for animating the movement of human respiration in 2004 [6] and here, we offer extensions that will follow from this initial work leading to more realistic physically based anatomical modeling aimed at breath training for patients.

Further, in medicine, extensive study has gone into very specific anatomical simulations particularly of the heart (hundreds are performing research in cardiac mechanics today) while other biological systems of importance and interest, such as respiration, remain practically untouched. One possible reason for this is the complicated nature of the process. While the heart is largely a "stand-alone" mus- 
cle, the respiration system is constructed of rigid bone and deformable muscle, cartilage, and tissue. All of these components must work together by actively contracting and passively responding appropriately in order to form breath. We plan to extend our investigations by creating physiologically valid, mixed rigidbody/deformable models and this paper represents a step in the process. Here, within the scope of breath training, we excite the system using motion capture to be able to encode individual breath signatures onto the model (See Figure 1).

\section{Torso Simulation}

Our torso simulation is broken down into five main components: spine, ribcage, diaphragm, abdominal cavity, and clavicles - all are highlighted in Figures 2 - 4. We use a mixed combination of torque actuators, applied to the spine, and a Hilltype muscle model for muscle elements that we use in the ribcage, diaphragm, abdomen, and clavicles. The torso simulation contains: 40 rigid bodies and a deformable body for the abdominal cavity, 1100 Hill muscle elements and 72 degrees of freedom in the spine. We followed our previous techniques described in [6] with a few notable exceptions, for example previously we allowed no articulation in the spine and treat it as a single rigid body.

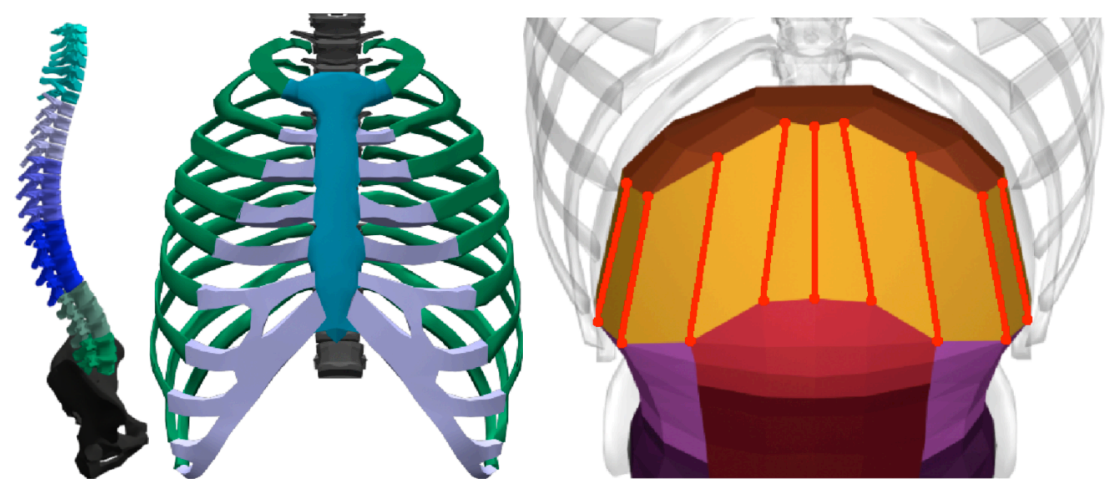

Fig. 2 Left: The torso simulation spine colored to denote sections. Center: The ribcage and sternum, also denoted by color. Right: Bright red line segments highlight the contractile muscles of the diaphragm.

Spine. The actual human spine is composed of 24 vertebrae in three separate sections: 5 in the lumbar, 12 in the thoracic, and 7 in the cervical. Laughter often includes noticeable movement in the spine due to the violent, involuntary contractions of the abdomen [24]. After a number of empirical tests conducted in our study of laughter, we concluded that combining groups of vertebrae gives the articulation we desire along with reasonable stability at a manageable timestep. In particular, as seen in the coloring of Figure 2, we break up the lumbar into two 
sections (L1-L3 and L4-L5,) the thoracic into three sections (T1-T4, T5-T8, T9$\mathrm{T} 12$,) and the cervical into two sections (C1-C3, C4-C7.) In addition, the skull is added as a separate rigid body connected to the $\mathrm{C} 1-\mathrm{C} 3$ section. The spine is rooted at the pelvic girdle and each joint is treated as a ball joint. Back stability muscles are accounted for by proportional-derivative (PD) servos.

Ribcage. The ribs are each rigid bodies attached to the spine via ball joints. The sternum and cartilage complete the ribcage as shown in Figure 2. We treat the portions of the cartilage as rigid, rather than approximating them as a more realistic, very stiff but deformable material. The reasoning for this is largely practical to avoid the stiff deformation which would immensely slow down the computation time of the simulation. We use universal joints to attach the cartilage to the sternum to avoid creases in those joints. Unlike previously, we add degrees of freedom in the connections between the sternum and cartilage so that we can generate a much wider range of motion for the ribcage.

Diaphragm. Our diaphragm is quite novel in contrast to previous approaches because we explicitly model the central tendon (shown in rust color at the top of the diaphragm in Figure 2) The diaphragm, which is shaped like an inverted bowl, contracts largely along the 'sides' of the bowl while the central tendon at its base sees much less (passive) stretching or contraction. Our diaphragm is modeled so that the muscle elements are aligned in the direction of contraction making the diaphragm much more effective as a "pump" for the air in the lungs.
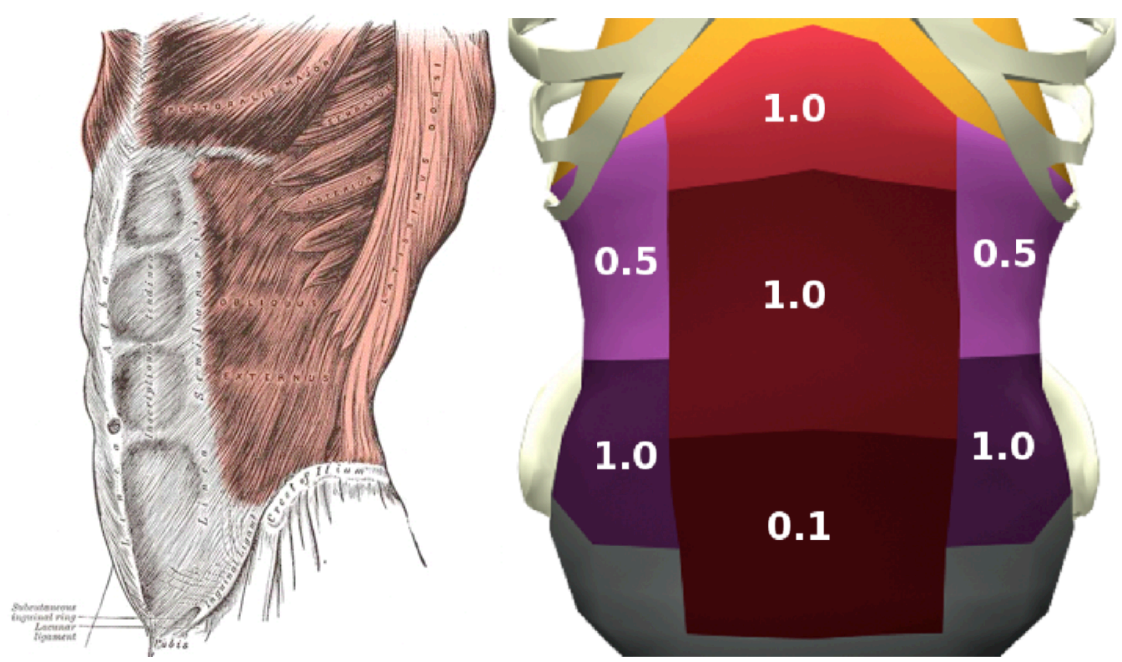

Fig. 3 Left: The obliques externus abdominis [3]. Right: The proportions shown are ratios of activation for the colored regions of the abdomen adapted from [4].

Abdominal Cavity. Our deformable abdominal cavity follows our previous design and methods by including a volume preservation method. In many forms of paradoxical breathing, activation of the abdomen is very important. According to 
[4], the pattern of activation of the abdomen is not uniform. We use their findings to identify five regions in the abdomen (shown in multiple colors in Figure 3) that we activate proportionately, as shown, based on their findings.

Clavicle. In many stress-related cases, shoulder breathing is prevalent. In our experiments, we include movement of the clavicle, which effects the shoulders and subsequently, the arms. Our clavicle is attached at the front of the ribcage close to the top of the sternum using a ball joint. To capture the interplay of the clavicle, the ribcage, and the spine we attach a fairly extensive set of muscles that have proper origin and insertions between these subsystems (see Figure 4). These muscles propagate activity between the components. In addition, we can use their contraction to control and add additional movement to the clavicle.

In many stress-related cases, shoulder breathing is prevalent. Therefore, in our refined torso simulation, we include improved movement of the abdomen and clavicle, which effects the shoulders and subsequently, the arms.
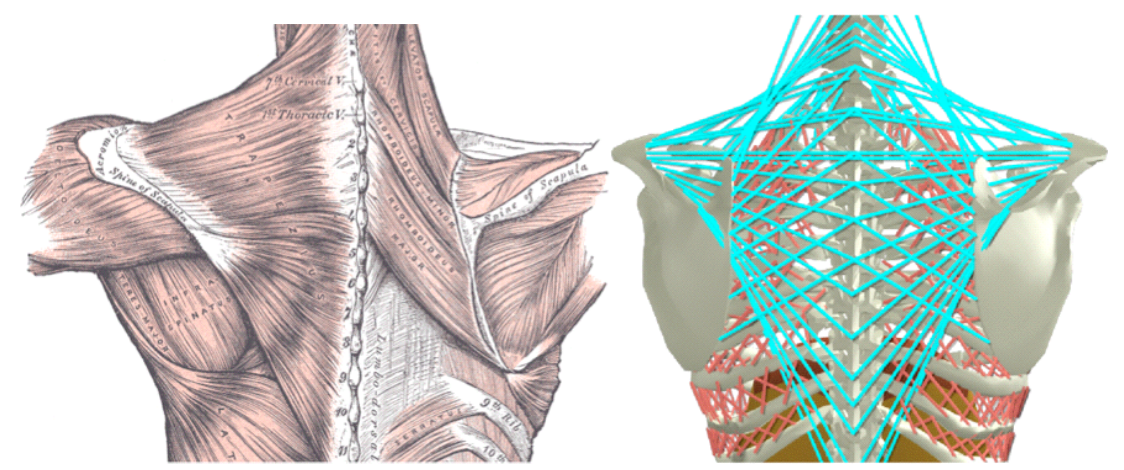

Fig. 4 Left: Anatomical shoulder muscles [3]. Right: Torso simulation shoulder muscles.

\section{Control}

To control the torso simulation, we propose a three-tier hierarchical controller. At the lowest level we compute forces using Hill muscle elements for the respiratory muscles, the abdomen, and the muscles attached to the clavicle. At the midlevel, we group like muscle components and activate them in unison. For example, all the muscle elements in the diaphragm receive the same inputs, even though each is applying a different force as appropriate to its specific conditions. At the highest level, we generate control signals based on the high-level characteristics of the motion. We apply force through each of the muscle elements using a Hill muscle model. Our formulation is derived from insights drawn from previous research on Hill-type muscle modeling, particularly [5]. The basic structure of the muscle model includes a passive element which contributes forces when the muscle is 
stretched and the contractile element which contributes force when the muscle is activated. For details regarding our Hill muscle model, we refer interested readers to see [24]. PD servo control of the spine allows additional control for full-torso bending as seen in the figure below.
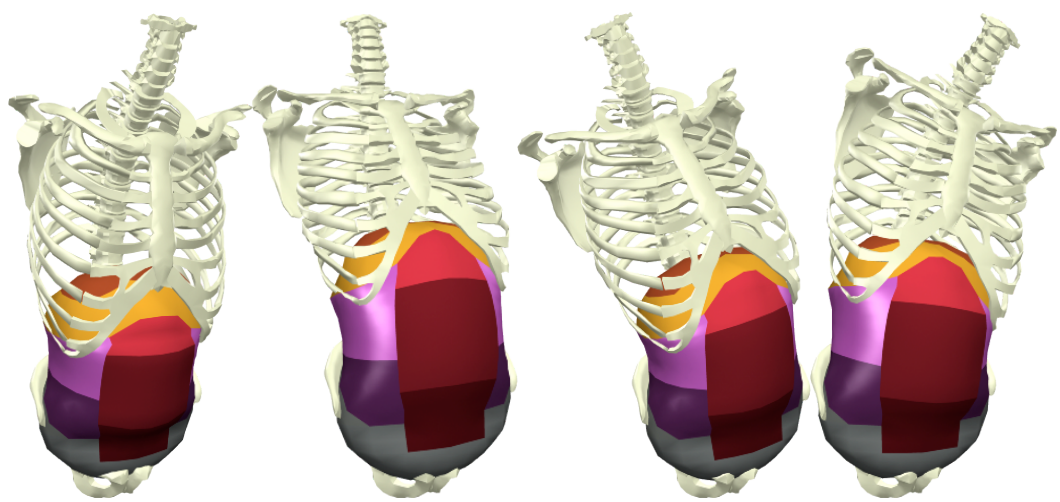

Fig. 5 Flexion, extension, and lateral bending (left and right) of the spine.

Functionally, the control that drives breath is generally split between the two moving subsystems, the intercostals and the diaphragm. These parts move in a synchronized manner and do affect each other but have unique control input based on their own neural activations. This split is reflected in the control of the humanrespiration system with the nerves which supply contraction input to the muscle groups stemming from different portions of the spine [25]. Further, during effortless breathing, only the diaphragm may be activated, without any intercostal contraction. We control activation for muscle groups by changing the contraction level over time for the muscle elements in the intercostals and the diaphragm. In Figure 6, we show that the result of simple periodic contraction levels for activation that are able to synthesize breath styles with quantitative similarities to actual human breath results

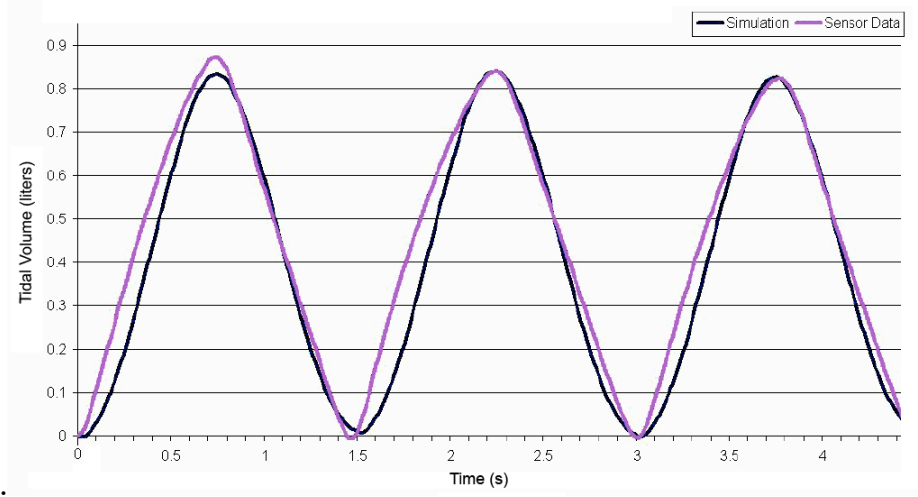

Fig. 6 Tidal volume comparison between simulation and air-flow sensor from a human subject. 


\section{Driving with Human Motion}

Motion capture data, recorded measurements taken from human subjects, faithfully reproduces many movements of the human body. We use a marker-based Vicon motion capture system to measure chest and stomach deformations using an ample suite of markers. In addition, the placement of these markers corresponds to anatomical landmarks such as the sternum, clavicle, and navel. The system is capable to track reflective markers attached to the body with a $3 \mathrm{D}$ accuracy within one millimeter.

Of course, the motion recorded only captures the gross external effects of the body. Seeing internal effects, such as the diaphragm is not possible without inference. For the purposes of visualizing anatomy for humans, captured data is no valuable because it is not possible to record the movement of the internal organs and muscles directly. In contrast, simulation holds the promise of realism and generality because it is based on the physical laws that govern the real world. However, motion created through simulation requires control in order to perform coordinated behaviors realistically. Our assumption is that given the breath motion data, i.e. only $3 \mathrm{D}$ points, we can generate a visualization that is the integration of the breath simulation with the marker data using the data to determine control for specific recordings.

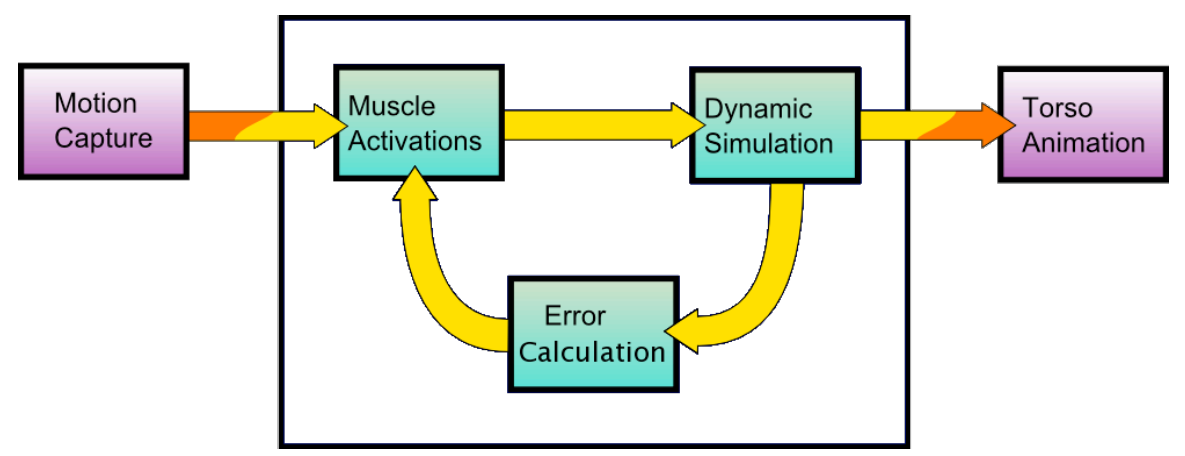

Fig. 7 An overview of the optimization process.

Optimization. Motion data cannot be directly used to control the simulation. However, we can determine the activation levels required to achieve the desired motion through optimization. We chose to activate the main muscle groups that contribute to respiration: the outer intercostals and diaphragm which are used for inhalation and the inner intercostals and abdomen which are used for exhalation. Although the abdomen is broken up into sections, we determine a single value for their collective activation based on the findings in the literature [4] and use it to compute the specific activation levels of each section as described. To further simplify, we link the timing parameters of the outer intercostals with the diagram and the inner intercostals with the abdomen. For the four different muscle groups, 
we only allow the optimizer to choose the maximum muscle activation for each muscle group. Thus, the optimizer has only four parameters to optimize over. Despite having a small number of parameters, the three-tiered hierarchy in place serves as the basis for the visually rich, complex motions produced from our system when acting upon the optimized parameters. We experimented with activation inputs such as sinusoids, step function and linear interpolation of control points, along with different parameter controls such as phase and period. We found that piecewise-linear sawtooth inputs produced the most visually pleasing results and come closest to the input data of different types of subjects.

Rather than optimizing for an entire breath sequence simultaneously, we use an idealized (average) cycle of breath from each patient. This "breath signature" is derived by analyzing characteristics of motion data. This trade-off allows computation of a small set of parameters in exchange for more specific optimizations applied to each breath cycle. It also allows for motion-cycling when the patient views her/his own breath. By placing virtual landmarks on the simulation, the system 'records' virtual motion capture data (from the simulation), thereby creating a one-to-one comparison of the simulation and the human. We use OPT++'s [26] parallel direct search method, which is an optimization technique that does not use derivative information to solve for the parameters. We used this method to take advantage of the parallelism in OPT++ since the function evaluation takes a considerable amount of time due to the simulation time of the torso system. Our cost function is defined as the error between the human and simulated motion data.
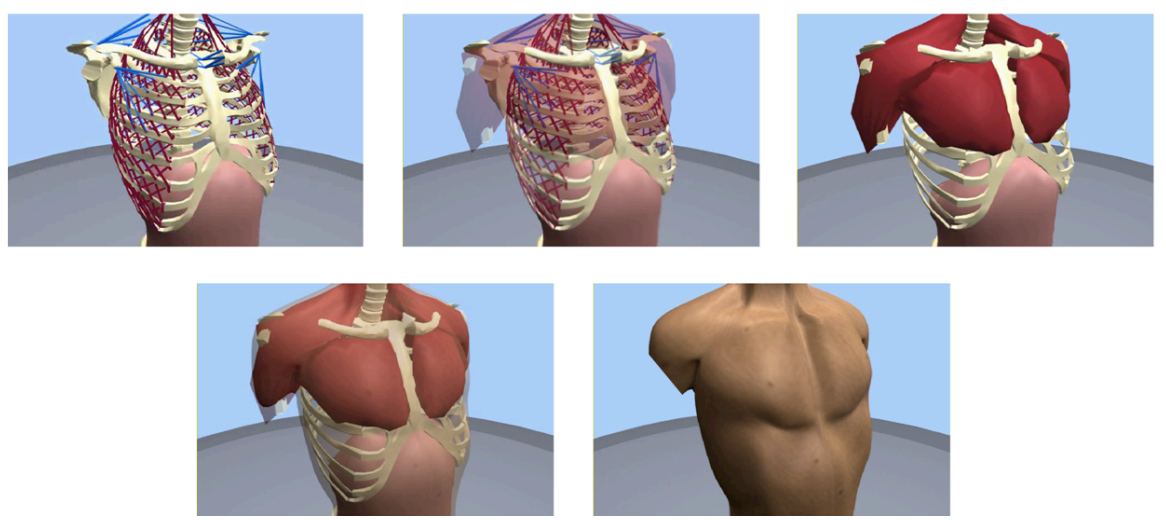

Fig. 8 Layers of the torso model used for demonstrating breath simulation.

The result is a motion synthesis that replicates the patients own motion. The motion can be viewed transparently, so that the patient and doctor can observe the internal function. Also, the relationship between the components can be seen. An example of the variety of visualizations possible appears in Figure 8. We expect 
that doctors will be able to control the transparency of the layers based on the demands of the situation.

\section{Results and Analysis}

We recorded the breathing behavior of four subjects of varying height and weight using a motion capture system. After recording the subjects, we determine their breath signature. The marker locations from the motion capture system are matched with our torso simulation. Then the optimizer iteratively tests a number of samples of breath control parameter sets based on the specified constraints. The system calculates the average displacement error, error $_{p}$, and velocity error, error $_{v}$, between the motion capture markers and each synthesized run of the torso simulation. We define

$$
\operatorname{error}_{p}=\frac{\sum_{0<i<m} \sum_{0<j<n} d(i, j)}{n^{*} m}
$$

where $m$ is the number of frames, $n$ is the number of motion capture points, $d(i, j)$ is the distance between the motion capture marker and the torso simulation marker. We define

$$
\text { error }_{v}=\frac{\sum_{0<i<m} \sum_{0<j<n} v(i, j)}{n * m}
$$

where $v(i, j)$ is the instantaneous velocity difference. Table 1 shows the results of the best solution found by the optimizer for each subject as well as the activation chosen by the optimizer. An interpretation of the values of the subjects allows us to make high-level assessments regarding individual breathing style based on the variability in activation between the subjects. Subject 1 primarily uses the ribcage with slight use of the diaphragm during inhale and a small amount of activation of both the ribcage and abdomen in exhalation. Subject 2 only uses the ribcage to inhale and no muscle activation in the diaphragm. For exhalation, this subject passively exhales. In contrast, Subjects 3 and 4 use an even mix of ribcage and diaphragm activation to inhale, while only Subject 3 uses muscle activation to exhale. Our system was able to capture these different types of breathing with an average displacement error of less than $7 \mathrm{~mm}$ per marker and an average velocity error around $6 \mathrm{~mm} / \mathrm{s}$ (See Figure 9).

Paradoxical Breathing. A common paradoxical breath pattern takes the form of contraction of the abdomen during inhale. We use our system to analyze this dys- 
function. We compared normal breathing with a simulation of this type of paradoxical breathing using tidal volume. We construct a paradoxical breathing example which was verified by our breathing expert, (co-author) Dr. Bakal. We compare this simulation to the same example without the activation of the abdomen. The results of our experiment can be seen in Figure 9 and 10. We can see that during paradoxical breathing, the simulation determined the tidal volume to be approximately $400 \mathrm{ml}$. If we remove the abdomen activation, the simulation determined the tidal volume to be approximately $800 \mathrm{ml}$. Clearly, there is a loss in tidal volume when someone breathes in this paradoxical manner compared to normal breathing. Figure 9 (right) shows a few comparison frames between the normal breathing simulation (top) and the paradoxical breathing simulation (below). The benefit of contracting the diaphragm is absent during the paradoxical breathing while it is used prominently in normal breathing. This observation argues for the use of a simulation such as our model since the effects of the diaphragm movement can not be seen from the exterior of the trunk.

Table 1. Table showing the average positional and velocity error between the motion capture markers and the simulation markers for the best optimized run for each subject. The activation levels for the outer intercostals, diaphragm, inner intercostals, and abdomen are shown.

\begin{tabular}{ccccccc}
\hline & $\begin{array}{c}\text { error }_{p} \\
(\mathrm{~mm})\end{array}$ & $\begin{array}{c}\text { error } \\
(\mathrm{mm} / \mathrm{s})\end{array}$ & outer & diaphragm & inner & abdomen \\
\hline Subject 1 & 5.66 & 4.64 & 0.305 & 0.040 & 0.018 & 0.007 \\
Subject 2 & 8.40 & 9.29 & 0.488 & 0.002 & 0.002 & 0.002 \\
Subject 3 & 8.62 & 6.17 & 0.254 & 0.099 & 0.033 & 0.010 \\
Subject 4 & 4.48 & 4.11 & 0.261 & 0.106 & 0.000 & 0.000 \\
Average & 6.79 & 6.05 & & & & \\
\hline
\end{tabular}

In our next step, for future work, we plan to begin to look into the recording of a patient's exact body to match physique. Techniques that allow this have already been reported in computer graphics [27]. In addition, it is critical that we investigate methods for validation of our model so that it can be viewed, especially by medical personnel with more confidence. Currently, failure for the optimization is possible but could act as an indicator of an unusual circumstance. One viewpoint is that rather than being construed as a failed optimization, a poor fit between our model and a subject's data may be an indicator of an anomaly - that something out of the ordinary is taking place and/or the patient's breath is truly exceptional. In 
such a case, further testing could be prescribed, including MRI or the like.
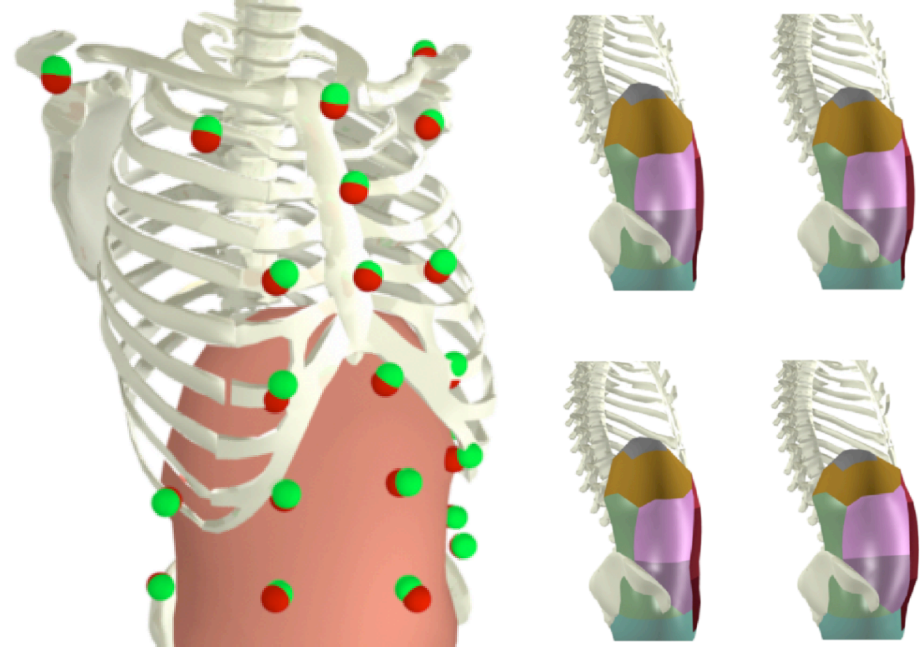

Fig. 9 Left: Comparison of the motion capture and simulated markers overlayed on the torso simulation. Right: Diaphragm comparison between the simulated paradoxical breathing (top) and normal breathing (bottom).

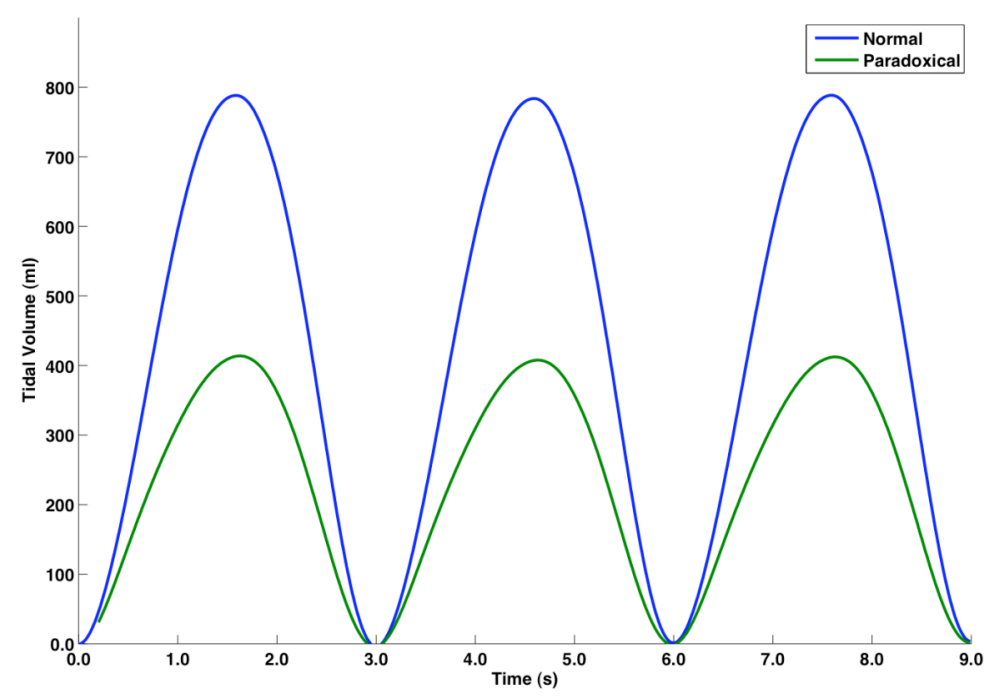

Fig. 10 Tidal volume comparison between normal and paradoxical breathing.

Conclusions. Our research is still preliminary. However, even if we see a component of failure in being able to personalize our respiration model (i.e. the riskiest 
thrust of the research) we expect the respiratory modeling will be of grand benefit. In addition to the novel and important testbed formed by breath training, simulation of respiration required a substantive understanding of the modeling. That said, we have made several tests which reveal our model is indeed quite realistic. Quantitatively, the tidal volumes and pressure results of our models are already quite close to the real human system. This is not surprising given the care and attention we placed on our selection of parameters for our system: our model matches the anatomy of respiration very closely. For example, upon cursory inspection, the skeletal anatomy is close to the real human in size, and muscle insertion and origins are accurate and our muscle activation using the Hill-type muscle model is very progressive by any standard. Including more aspects and input from respiration experts will undoubtedly improve this trend.

In addition, based on anecdotal evidence, we already anticipate an easy acceptance of the generic respiration system as a tool for visualizing and training breath. Qualitatively, breathing with the system is 'natural', i.e. synchronizing breath with the animation has been an unsolicited trend among observers. Several viewers upon their first time viewing of the animations, begin to align their breath with the animated breath without any suggestion or indications offered to do so. That is, they involuntarily begin to 'breathe with' the simulated motion. This is an exceptional phenomena because it is exactly what is needed for breath training. If the motion capture matching does not reach fruition, we will develop pure simulations and based on our experience, we believe that patients will readily match breathing, especially if encouraged to do so.

\section{References}

1. Kaye J, Metaxas D, Primiano Jr F.: A \#d virtual environment for modeling mechanical cardiopulmonary interactions. In: CVRMed, pp. 389-398 (1997).

2. Promayon E, Baconnier P, Puech C.: Physically-based model for simulating the human trunk respiration movements. In: Lecture Notes in Computer Science, 1205, pp. 379-388 (1997). Springer Verlag CVRMED II-MRCAS III first joint conference.

3. Gray H.: Anatomy of the Human Body. Lea \& Febiger (1918).

4. Hoit J, Plassman B, Lansing R, Hixon T.: Abdominal muscle activity during speech production. In: Journal of Applied Physiology, 65(6), pp. 2656-2664 (1988).

5. Zajac F.: Muscle and tendon: Properties, models, scaling, and application to biomechanics and motor control. In: CRC Critical reviews in biomedical engineering, 17, pp. 359-411 (1989).

6. Zordan V B, Celly B, Chiu B, DiLorenzo P C.: Breathe easy: model and control of simulated respiration for animation. In: ACM SIGGRAPH/Eurographics symposium on Computer animation, pp. 29-37 (2004).

7. Lee S, Terzopoulos D.: Heads up!: biomechanical modeling and neuromuscular control of the neck. In: ACM Transactions on Graphics, 25(3), pp. 1188-1198 (2006).

8. Platt S, Badler N.: Animating facial expression. In: Computer Graphics, pp. 245-252 (1981).

9. Waters K.: A muscle model for animating three-dimensional facial expressions. In: Proceedings of SIGGRAPH 87, pp. 17-24 (1987). 
10. Terzopoulos D, Waters K.: Physically-based facial modelling, analysis, and animation. In: The Journal of Visualization and Computer Animation, pp. 73-80 (1990).

11. Lee Y, Terzopoulos D, Walters K.: Realistic modeling for facial animation. In: Proceedings of SIGGRAPH '95, pp. 55-62 (1995).

12. Kähler K, Haber J, Yamauchi H, Seidel H.: Head shop: Generating animated head models with anatomical structure. In: Eurographics/ACM SIGGRAPH Symposium on Computer Animation, pp. 55-64 (2002).

13. Sifakis E, Neverov I, Fedkiw R.: Automatic determination of facial muscle activations from sparse motion capture marker data. In: ACM Transactions on Graphics, 24(3), pp. 417-425 (2005).

14. Gourret J, Magnenat-Thalmann N, Thalmann D.: Simulation of object and human skin deformations in a grasping task. In: Proceedings of SIGGRAPH 89, pp. 21-30 (1989).

15. Albrecht I, Haber J, Seidel H.: Construction and animation of anatomically based human hand models. In: Eurographics/ACM SIGGRAPH Symposium on Computer Animation, pp. 98-109 (2003).

16. Tsang W, Singh K, Fiume E.: Helping hand: An anatomically accurate inverse dynamics solution for unconstrained hand motion. In: 2005 ACM SIGGRAPH/Eurographics Symposium on Computer Animation, pp. 319-238 (2005).

17. Chen D, Zeltzer D.: Pump it up: Computer animation of a biomechanically based model of muscle using the finite element method. In: Proceedings of SIGGRAPH 92, pp. 89-98 (1992).

18. Scheepers F, Parent R, Carlson W, May S.: Anatomy-based modeling of the human musculature. In: Proceedings of SIGGRAPH 1997, pp. 163-172 (1997).

19. Wilhelms J, Gelder A.: Anatomically based modeling. In: Proceedings of SIGGRAPH 1997, pp. 173-180 (1997).

20. Nedel L, Thalmann D.: Real time muscle deformations using mass-spring systems. In: Computer Graphics International (1998).

21. Teran J, Blemker S, Ng-Thow-Hing V, Fedkiw R.: Finite volume methods for the simulation of skeletal muscle. In: Eurographics/ACM SIGGRAPH Symposium on Computer Animation, pp. 68-74 (2003).

22. Teran J, Sifakis E, Blemker S, Ng-Thow-Hing V, Lau C, Fedkiw R.: Creating and simulating skeletal muscle from the visible human data set. In: IEEE Transactions on Visualization and Computer Graphics, 11(3), pp. 317-328 (2005).

23. Pratscher M, Coleman P, Laszlo J, Singh K.: Outside-in anatomy based character rigging. In: 2005 ACM SIGGRAPH/Eurographics Symposium on Computer Animation, pp. 329-338 (2005).

24. DiLorenzo, P, Zordan, V, and Sanders, B. Laughing out loud: Control for modeling anatomically inspired laughter using audio. ACM Transactions on Graphics, SIGGRAPH Asia, 27(5):125:1-125:8, 2008.

25. A.H. Mines, Respiratory Physiology, Raven Press, New York, 1993.

26. Meza J, Oliva R, Hough P, Williams P.: OPT++: An object oriented toolkit for nonlinear optimization. In: ACM Transactions on Mathematical Software, 33(2):12 (2007). Article 12, 27 pages.

27. Allen B, Curless B, Popovic'.: The space of human body shapes: reconstruction and parameterization from range scans. In: ACM Transactions on Graphics, 22(3), pp. 587-594 (2003). 